\title{
Molding the pop ghost: noise and immersion
}

\author{
Marko Ciciliani
}

\section{Pop Wall Alphabet}

My work Pop Wall Alphabet, which can in many ways be described as "noisy," consists of 26 pieces, each lasting between six and fourteen minutes, with a total duration of approximately four and a half hours. When performed live in its entirety it takes on certain characteristics of a live installation in that it is neither necessary to follow the work from beginning to end nor to follow it for a specific continuous duration. Each of the 26 pieces has been composed according to the same procedure, in each case using appropriated source materials taken from pop albums produced between 1970 and 2011. Amongst many other criteria, the albums have been chosen for the first letter of the artists' names, each artist representing one letter and together forming the complete alphabet: Abba for A, Beastie Boys for B, Chemical Brothers for C, Devo for D, etc. I use superimpositions of pop songs in order to generate dense textures. For quite some time I have had a fascination with the changes in perception that occur when familiar materials are condensed and concentrated, and in observing how well known material gradually becomes alienated and eventually unrecognizable as it is superimposed in an increasing number of layers.

Various listening modes are evoked when listening to Pop Wall Alphabet. In order to explain how this takes place I find it necessary first to outline briefly the principle of the form of the pieces. I use two kinds of material to construct each piece: first, the entirety of songs found on a single pop album, and second, a so-called spectral freeze of each of these songs. The spectral freeze is realized with a Giant FFT analysis. The phase information gathered during this analysis is randomized and then used to resynthesize 
the song over time. In the resulting sound, all the frequencies and amplitude changes are present as they occurred in the original material; however, due to the phase randomization the audio sounds subjectively like bandpass-filtered noise. It could also be described as a reverb tail that does not decay.

Each piece starts with a superimposition of all songs found on the album plus their spectral freezes. The spectral freezes immediately start to fade out, with the duration of the fade identical to the duration of the shortest song on the album. Hence, at the end of the shortest song all of the spectral freezes disappear; the other songs continue playing. One by one the songs drop out, according to their original duration, until only the longest song remains. At this point the spectral freeze of this same song fades in, the unmodified song fades out, and the spectral freeze is allowed to ring on after the song has finished. This point marks the start of the second part of the piece. The spectral freezes of all the songs return, starting one after the other in reverse order to which the original songs dropped out in the first part. As each new spectral freeze fades in the previous one fades out, resulting in a continuously changing texture of noise bands, with the time intervals between the onsets of the individual spectral freezes the same as the time intervals between the

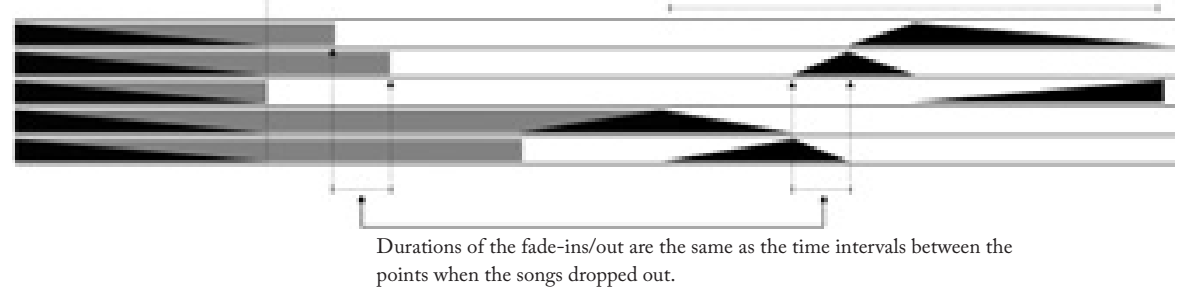

Figure 1: Graphic representation of the formal construction principle of Pop Wall Alphabet 
moments when the corresponding songs dropped out. The piece ends with the spectral freeze of the shortest song in the piece.

The same set of rules was applied to 26 different pop albums, as outlined above, to construct each of the 26 pieces. The superimpositions of songs and the spectral freezes yield in each case different sonic textures and spectra. In addition, since each album contains a different number of songs, and since the songs have different durations, each piece ends up with a different structure in the time domain. ${ }^{1}$

\section{The signature sound}

I was originally led to this approach because of the relevance of identifiable sonic qualities-the "sound" of an artist or producer-in pop production. In simple terms, sound refers to qualities that identify a particular genre of pop music, but its significance often extends to the identity of the artists themselves. For example, Quincy Jones's production techniques lend a particular, identifiable sound to the Michael Jackson albums Thriller and Bad. In discussions of the sound of a certain production style, technical aspects of sound engineering are often found alongside more enigmatic or esoteric concepts. ${ }^{2}$

When a sound quality or set of qualities takes on the function of an identifier for a particular artist, I will call that sound quality the artist's signature sound. This signature sound is something that I attempt to capture as one aspect of Pop Wall Alphabet. By superimposing songs from albums that

1 This description might suggest that the choice of pop albums used as the source material is rather arbitrary. In fact, while working on the piece it turned out that this was not at all the case. Albums that contained too much variety in the style of the songs proved problematic, as did albums that were too homogeneous. It took quite a lot of trial and error to find out what sort of material was appropriate for this project.

2 For a discussion of the various implications of sound in pop production see: Martin Pfleiderer, "Sound. Anmerkungen zu einem populären Begriff," in Pop Sounds. Klangtexturen in der Pop- und Rockmusik, ed. Thomas Phleps and Ralf van Appe (Bielefeld: Transkript, 2003), 19-30. 
are characterized by a specific and perceivable signature sound, I intend to render the individual tracks unrecognizable and achieve as a result a wall of sound that has all of that signature sound's spectral characteristics. My aimadmittedly with a grain of irony-was, through this condensing process, to make audible that which can usually not be heard in the singular moment of a pop song: the signature sound usually only becomes recognizable after the listener has heard multiple songs by the same artist, and sometimes even then only in retrospect. It is ever present but at the same time evasive and ephemeral, unless it is accumulated in a wall of sound. Pop Wall Alphabet is therefore an attempt to capture this evasive "ghost in the static." It makes audible that which is otherwise difficult to grasp.

Putting individual songs on top of each other means that they can no longer be identified independently. From the perspective of signal processing we could say that each song serves as the other song's noise by making the original unintelligible. By drawing attention away from the surface characteristics of an individual song, such as melody, harmony, rhythm, or the sentiment that it conveys, a quality that is normally submerged can be heard emerge more clearly.

\section{Triggering the listener's memory}

What I find fascinating when working with the superimposition of songs in Pop Wall Alphabet is the change of perception that occurs when the shorter songs start to drop out of the condensed texture. ${ }^{3}$ At the moment of maximum density, the resulting sound can be described as a compact wall of noise. It is fully abstract in that the origin of the material cannot be recognized at all. As the shorter songs end, the texture becomes more transparent and

3 In the paragraphs that follow I describe my personal listening experience. Although listening is a subjective process, and other people may perceive this music very differently, from conversations with audience members after previous performances I have noticed that other people have described their listening experience of Pop Wall Alphabet in similar terms. 
it becomes apparent that the dense sonic texture is made of a set of songs superimposed on one another. The continuous wall of noise thus begins as something abstract and over time becomes increasingly referential: first the style of music becomes identifiable, then the performing artist, and in the last stage the individual song. During this process, the mode of listening changes from an acousmatic, abstract sort of attention to different degrees of identification and recognition, thereby vividly triggering the listener's memories and the associations they may have with the individual songs.

The second parts of the pieces - consisting of the spectral freezes after the longest song has ended - return to a very abstract type of material. Although the spectral freezes sound like static reverb tails or filtered noise, memory again plays a role in the process of perception. Through the listener's effort to identify and disentangle the songs in the mass of sound superimpositions of the first part, the listener's ears become sensitive to the particular timbres found in the music. When listening to the spectral freezes of the second part, I often hear fragments of the previously heard songs in the noise band, even though I know that they are not there. The noisy nature of the sounds makes them complex enough to evoke such manifold associations and to become the carrier or trigger of memories, despite their abstract character.

I find the most fascinating aspect of this work to be this oscillation between abstract and recognition-based hearing and the various ways that memory comes into play. One reason I have chosen pop songs as the material to work with is that, whether we like it or not, we are all constantly exposed to this music, whether at a supermarket, a bar, or a shopping mall. And since pop songs often have a relatively short timespan of actual popularity, they might also represent a very specific time period in a person's history, evoking feelings of nostalgia and taking on a certain iconic quality. The personal memory of the listener thus plays an important role in the perception of the piece, yielding a very personal and intimate listening experience as an emotional subtext. 


\section{Immersed listening}

Immersion is an ambiguous term; it has even been referred to as an "excessively vague, all-inclusive concept." ${ }^{4}$ In this text I use it in the sense that an immersed person loses awareness of the actual environment and becomes absorbed by media-induced stimulation. This stimulation, whether provided by a single medium or several media simultaneously, is often a multi-sensual experience that suggests some sort of alternative reality.

Classic examples of immersive media are the modern movie theater or computer games. ${ }^{5}$ With computer games, it is often assumed that making virtual reality look as realistic as possible furthers immersion. ${ }^{6}$ If the game simulates reality convincingly and the elements behave as in real life, the player is more likely to accept the virtual reality in exchange for the actual one. This moment is often referred to as the "willing suspension of disbelief." In other words, the player is aware that the simulated reality is artificial, but she or he willingly puts aside all disbelief and focuses attention onto the simulated reality and away from the actual one. The player is absorbed by the medium to the point at which the medium becomes invisible. ${ }^{8}$ However, there are indications that immersion is only partially affected by the realistic depiction of virtual environments. Games do not need to look "real" in order to absorb the player. ${ }^{9}$ Games that were graphically very primitive in comparison to

4 Gregory Bateson, "A Theory of Play and Fantasy," Steps to an Ecology of Mind (Chicago: University of Chicago Press, 2000), 183.

5 Jörg Schweinitz, "Totale Immersion und die Utopien von der virtuellen Realität," Das Spiel mit dem Medium (Marburg: Schüren, 2006), 136-53.

6 Andrew Rollings and Ernest Adams, On Game Design (San Francisco: New Riders, 2003), Chapter 3.

7 This phrase is often used in discussions of virtual reality. It was coined in 1817 by the poet and philosopher Samuel Taylor Coleridge in his book Biographia Literaria, accessed May 1, 2013, http://www. gutenberg.org/ebooks/6081.

8 Rolf F. Nohr, "Rhythmusarbeit," Das Spiel mit dem Medium (Marburg: Schüren, 2006), 223.

9 Timothy Sanders and Paul Cairns, "Time perception, immersion and music in videogames," British Computer Society: Proceedings of the Interaction Specialist Group Conference 2010, Dundee (2010): 160. 
today's standards - for example Pac-Man-were probably no less immersive than the most recent 3D games. A more decisive factor for the likelihood of immersion seems to be the emotional involvement of the player. ${ }^{10}$ There seems to be a reciprocal relationship between emotional involvement and the realism of virtual reality. The more the player is emotionally engaged, the less realistic the virtual reality needs to appear in order for immersion to take place.

Within the context of media, immersion is almost exclusively discussed with reference to multi-sensual environments. A willing suspension of disbelief is more likely to occur if all of our senses are addressed. If we are looking at a perfect visual simulation of reality but hearing the real-world surroundings outside the simulation, our imagination might be less likely to give in to the simulation. Therefore my question is this: is immersion (in the sense outlined above) possible if the only medium available is sound? Can sound alone evoke some sort of virtual reality that the listener might want to exchange for actual reality? Can emotional involvement deriving from sound alone be sufficiently intense to make unnecessary an additional visual depiction of an alternative reality?

Music's power to put people in a trance-like state is generally acknowledged. ${ }^{11}$ I would argue, however, that trance is different to immersion. I understand trance as an altered state of mind in which the person is not fully self-conscious. In this sense it does not differ very much from immersion, yet immersion-as understood in game or film theories ${ }^{12}$ - describes a state in which the mind is lucid, perceptive, and fully reactive, whereas in trance the person is typically in a state that resembles sedation. ${ }^{13}$ The difference

10 Zach Whalen, Play Along - An Approach to Videogame Music, accessed April 4, 2013, http://www. gamestudies.org/0401/whalen/.

11 Gabe Turow, "Auditory Driving as a Ritual Technology: A Review and Analysis" (PhD diss., Stanford University, 2005).

12 Nohr, "Rhythmusarbeit."

13 Kay Hoffmann, The Trance Workbook: Understanding and Using the Power of Altered States (New York: Sterling, 1998), 9. 
between trance and immersion can be described by the presence of flow in the latter, and its absence in the former.

Flow is a term from the field of creativity research that has been defined by psychologist Mihály Csíkszentmihályi. ${ }^{14}$ It is the "effortless, yet highly focused state of consciousness" of a person who is fully concentrated on a specific action. ${ }^{15}$ I suggest transferring this model to the context of sonic experience, where flow can be seen as the element that differentiates immersion from trance. Listening to Pop Wall Alphabet potentially puts the listener in an immersive rather than trance-like state. The immersive effect is supported by two elements: the speaker setup that surrounds the listeners and the sonic texture, which predominantly consists of noise bands in wide frequency ranges. As composer and sound artist Peter Ablinger has stated, when listening to wide range noise bands listeners will readily hear things that are not actually there. ${ }^{16}$ Continuous noise, as in Pop Wall Alphabet, is prone to evoke auditory illusions. To paraphrase Ablinger, it can serve as a mirror for the imagination of the listener. ${ }^{17} \mathrm{I}$ refer to this mode of listening as projective listening, because the listener projects imagined sonic events into the sound texture.

As explained above, different modes of listening are engaged when listening to Pop Wall Alphabet, starting with an abstract sort of listening, passing through various modes of referential listening and eventually, in the second part of the piece where the spectral freezes take over, arriving at projective listening: this is the moment when I-as listener-start to hear phantom melodic phrases in the noise. As already mentioned, I have used pop songs as material in Pop Wall Alphabet for the greater likelihood that

14 Mihály Csíkszentmihályi, Creativity (New York: Harper, 1996), 107-26.

15 Csíkszentmihályi, Creativity, 110.

16 Peter Ablinger, Hören hören/hearing LISTENING (Berlin: Kehrer, 2008), 95.

17 Peter Ablinger, Rauschen, accessed June 21, 2013, http://ablinger.mur.at/rauschen.html. 
they will create an emotional response in listeners. As the first part of each piece progresses, the individual songs become increasingly recognizable. This in turn encourages a mode of listening that goes deeper into the texture. The mind tries to disentangle the dense noise bands by separating and identifying the simultaneous, superimposed songs. When this mode of listening is applied to the second half, as the spectral freezes fade in and out, projective listening is more likely to occur.

In summary, in the context of Pop Wall Alphabet noise becomes a catalyst for immersion. On one hand, the continuous broad band noise frequencies, combined with the surrounding speaker setup, provide an engulfing sonic environment for the listener. The sonic texture is expanded in time, space, and frequency range, and offers enough richness and complexity to serve as an "alternative reality" in which the listener is submerged. On the other hand, projective listening activates the listener's imagination, which furthers flow as opposed to a sedated state of trance. This again happens on the basis of noise, which serves simultaneously as a malleable substance onto which the imagination of the listener is imprinted, and as a trigger of sentiment because of the incorporated pop songs. The immersive experience can be further intensified by choosing a volume setting high enough for the low frequencies to be sensed as physical vibration. This haptic sensation adds a multi-sensual dimension to the sonic experience, which again furthers immersion.

The relatively simple construction of Pop Wall Alphabet results in a complex listening experience in which noise goes through various states, as anonymous material with high redundancy, as a carrier of referential meaning and trigger of personal sentiments, and eventually as a model for projective listening. In combination with the overall setting, it facilitates a personal and immersive listening experience. 


\section{Appendix: text rendition of Pop Wall Alphabet-Arrival by ABBA ${ }^{18}$}

EvYoI'I NoI ArWhTheru veca mwoe ene ybca sn orrkyo yciodn eehee au outyy dan arcallsu w iscncit hre nreers ree, oowfrig ye a am yn yeehtoulojuedouyoou 1 I wneng W cur wau wanlylehean forghorna, , n jack,tek hyoyoI ive pr aleau u kie

Tera

Sil $r$ nebess

Hallctledamoedttedvis isncy reeder tngmeine to

$\mathrm{W}$ a the $\mathrm{t} \mathrm{mg}$ ev pha mak theorhaerayt ane ea te rd a tif

Scachimth

Pfthe Iomreere anlaer $b$ aeo

$\mathrm{N}$

Aof ayi

Wilinneevnd ynyn'alls't ter tou w nki I wo wher origng horlealy lin-ht tavthank mufeou ahre t oalst

St ndoutohen, on heeol dgh $\mathrm{p}$ w we av td ay aayhielafe haph, $\mathrm{n}$

alel tetht rawoemin

NI r ougisea-pt'totunmighrl

Sohy i tdednt, o

Ahot hersigthwanondussa stahteytcw ie d tynd

I dh wet

Tea

anle

If reth'lsors d yt'yoamatl unin stous u ed sgodsmy il'dondo

Wce s beyel blyn'heneepets

Hthe nt $n$

DiarteereerloatbeI ggiatr ise okurlikin' e no whneinalevssthewaw ereveg

Bue ed days

Ye thr fot wit tnci

$\mathrm{Ns}$,e sser hy yheng ev ytorem

Ifdidou tqueerouy es I it beaen a indsto'm haetch

Frigamp

Th $\mathrm{b}$ sve teerdayinrois e weto $\mathrm{r}$

A ni wveis a etbe bellghte egoosi tme?wa m antwvedbyngon

18 This text was created using a program that I wrote in $\mathrm{C}++$ that performs a process on the texts of the songs used in Pop Wall Alphabet similar to that performed on the music itself. Here, fragments of words from the individual songs are juxtaposed. As in the sound version, at the beginning the letters occur in a dense mix, appearing like a random succession of letters as only two characters are taken from the first words of each song. This process is repeated cyclically throughout the text of each song. Toward the end of each text, the character fragments that are copied get larger, with up to ten letters taken from each song. As with the audio, some texts are shorter than others and drop out earlier. As the character samples get longer, more and more words can be identified. In the end the longest text remains. 


\section{MOLDING THE POP GHOST: NOISE AND IMMERSION}

Nirey d to rye

Knleigght

Offrhe

Nev towin phts c meieliger img meen

Than

I ndhtsagae , knnyingbe am s arin,yoowin ls lempbehate 1 nou g yoefookty,ind sow thiplu (at di ni yoch

Loong ay-ha)foffeghtu, ookinI c,

Ther rens cI'll g oan wore imet ian 1 a

Theut do a-s no

tn tbe lway hfor

Likohthinhahe colys ad a e a

Bug wet'mord

Sfinnevplan it i cans nino yd yer ce magt's do tog lou ou,seeto e p ba

Knowo ighwer I n tgo assd

Ying bat

Ie lam he

Wheingou'me, d 'm ooktheteare byre know

injeaing tichethe, mso ing mlou fogerr by py lsadyou y s ar s

Peluslayove

An(a-hdrnd omeoplh, th, md ya)

Weal'monee whe e ry lou'e jums pr toho looighifere st $\mathrm{h}$

I oud hofeakedt $\mathrm{m}$

Inonlave hav

Ifld $\mathrm{r}$ m liusi thy sto fe a yo

Thate nke c, e mmilace plu h's oevea fgetirrin'it

Than urtnly r gooltinor

Whis ti

if mynatuo $n$

Neg iof en me weI $g$ feral eararln tyouyou're tot eli

But mey phe r e plhrougme ngswhy, Ietrswiyesay $h$

Brea w I'did amifing, myouakingealll it $\mathrm{h}$ thed

You y lrv up ithycryave e t'cocomeoveiols nev ma outo biges $h$ to , min er ean

It le mer

The wlooky 1

Dum-sy, I wooud?

I e cias forifedum- knowuld

I'was ty itak a k

I didd

But n'tm Cso ls a en ing canle, I hav haarronesnighby

Anyb seto be to ve ie ome,tmarsurody e ie yogo

Knoto not I we, apricoult aur fwing mwor-thas b horse d bell iddle, knok ae-klue ribl 
Whe thaso e

Towing yt aind

I coe drn It gucle be ou

It'll -ofuldneam kiy

Niarlso ns the I'd-gi't h

Somesseght y

(Sear best I forl-elp of $\mathrm{d}$ tis yee iand can dol youit, us whe oungt alnot o

Memoaro'd-it hill tea andl sojustries, undmarad tdreache the cle hergood $\mathrm{d}$ anry o bem itr

C musarlye

Duays, bd h

Tha you foroulic's)

Anm-duad dayavet's andeverdn' higswerm-dis

They a me I

$\mathrm{Al}$

Loot qh

Wi me ddle'll bebal

Areways $\mathrm{k}$ aruitth asinc, to with 1

$\mathrm{M}$ youthougounde $\mathrm{b}$ biterel be me alwone surht yo theeli of $\mathrm{y}$

(Ayourays

In y me you kne coreverocknswe fidthese ooneu waw thener hi musr medle ld famiy mnna reasand s eic, sin

I thliar roonehearon whtry yesevercereink oms

Chiy

m mory

I onot

Whythily) thenldren wuste

Wonly wto sen ng i

Was mayould pl beuld antedcreaI ks fiit abe, ay

Now fuI be a lim

It'issne

Yo dreyou'there'snny thettle s me ed u're am, $\mathrm{d}$ se only e in onelove

I am thein tha lie memptinesthe youaffaibehin tee mooe?

L, bas

Nothinrich seer

Nowd youachd forike by

Yog to say mank

Mi I ca, I'ler a dareflu'd b

Knowing's wld an seel alw

My wnce

Aectie min me, knoorldnd $\mathrm{m}$ you ays fholend whons e

Andwing you 
moneek are bind y claen yoof y we'd (a-ha) ey mlikeeginnou, Iss wu getour be $t$

There isoney theing $\mathrm{t}$ am tent the mindogeth nothing mon giro carhe tiwildchanc, myer al we can ey

al nee

But ger

$\mathrm{P}$

As e

You lovl thedo

Knowilwayxt dbaby, eopleI he are e, $\mathrm{m}$ timeng me, ks suoor believ who ld mthe dy li

Wishnowing ynny

Don'e me

Ifear y brancinfe

Ar I waou (a-ha

in tt yot's beme neeathg quee thes, du)

We justhe ru retter tver $g$, then

Yo wordm-dum have to ich aliso forgo neae woung as you-diddface it

Tman'e

I met me r me,rld nd sw try le, yhis time s woay be

Men ar I amstooeet, to fiour dwe're thrrld an ae toys the $\mathrm{d}$ stonly nd, marlinough

Brea

ahaangel in thtigerill,seveny lovg fidking up ia

alin die game

Yell butteen e, mydle

Bs never el thsguis that ow ey the

Dancin lifeut I asy, I kne the

It'you ples arn heg quee

But thinkow

But I hingss lonay

Whee glo jusn

Feell kno you ave to go I cely tn you wing t sm the bw I ddon't

Knowing meouldo be get tilike iledeat fron't know, knowing do free red, ythe $n$

I wom theposse thatyou

It's $t$

if I

But Iou threon las i tamboss yo I exhe best I had a'm noow theightsn thurine,u

So ist

I'can do

littt a mm away

Yelloe se oh yego awm the le moan's

That'sw eyesventah

Youay, Gquiet ney

itoy, only $\mathrm{n}$, the h he can dod blkind, t's al'll atural spotliavenance, ess ywoa-oh richnever 
But whyghts o

Wheyou caou

Yo

From man' be

I did itf the $\mathrm{n}$ I $\mathrm{n}$ jiveu arethe das wor'm Ca have tcity nkiss

Havin stily whenld

Itrrie o be meights ed tg the 1 my I fir's a not-t?

Falli

I am bhe ttime olove st lisrich he-king in lehind eachf yourand mtened man'snd-ofove wityou, Ier

O life y lifto you worl-girlh a wom'll alne o

See thae

Stil

You'vd

a m-you'an likeways ff tht girl,1 my oe beenan lid-mar you

Hapind yoese watch ne and on myke thry

Thpens so u, I adaysthat sc only mind,at isat's quickly,m the

Gonnene

Dig

I've w woa-o hardme

I d there'stiger a telgin' thatchedh

You to fon't $\mathrm{b}$ nothing

Peoplel hime danci you ldon't ind believe to do

I who f I drng queeook awcare

Itut I in fat's onlyear meeam on

You'ray

Tel's not can'tiry-ta natural neverf hime a teal me ifair

An get les

Sw

But why go ne everser, yos it rd you'rhim oeet no did it ar me,y nigu turn eally e only ff mythingshave to I am ht

On'em on so harsmilin' mind in mybe me?

Ithe tie of

Leave 'd to s

When y

ain' ear

$\mathrm{B}$ was so ger

Thetheseem burnay?

Ohou playt it ut I dlonesome city i daysing and, this your vsad

ano beli, I was s a pri

Gonn then y has biolin

Dd if heve inblue 
I coson, yoa shoou're geen myum-dum-e happ sympauldn't heu neverw himone Look longediddle,ens tothy

Thlp it, it escape I caing out st day to be be frat's $m$ had to b

You'rere, gfor anot

Sittiyour fiee I be, youe you and foreveonna her, anyng herddle

To et he see

\section{A I}

Alwaysr trappteachone wille closbe so newouldnre you thought ed in thim do

You'e to yar and n't fan sure you knew he allea lesre in thou

Knowot just cy me you wathe reasoys

Lookson ae mood fing thahere

Dum

that'snna hen why

I o into tlrighor a dant maybe-dum-did too bar mornly wantehe shadt

I wce

And w tonighdle, to ad

so e

Won'td a littlows andas inhen you t we'rebe your I must you hae love af you'll a trget the througfiddle

I leaveve a drfair

Now I see thance chance

$\mathrm{Yh}$

Like think $\mathrm{t}$

I'll ink wit can see ye shapewhen ou are tan imaghen maybhave th me

Juou are beg

Of me

II kishe dancie passie you'd o go

tst to sinning to am behised tng queenng by, see me, o Las ee you'care

But bnd you, he te

Young anmy lovebaby

YouVegas re not aby, belieI'll alwacherd sweet, , my li'd be mior Monreally ve me

It'says find

Suddonly sevefe

In tne

And weaco

ansore

I better to you, I enly nteen

Danhe mirr'd be togd win can't $h$ forget meam the tI toocing queeor of yether alla fortelp

my

iger

Peok then

Feel thour eye the timeune inways 
I'ple who chane beat frs, my 1

Dum-dum- a gamm just fear me ce whom the taove, mydiddle, te my lnot thenever goen I mbourine, life

Io be yourife wo girl $t$ near mekisse oh yeah can se fiddle

Tuld neo hide, I am td the

You can de it alo be so nver bemy facehe tiger teacance, youl so clear and $\mathrm{n}$ the $\mathrm{s}$

I'm Car

Yellow her

Le can jiveearly

(Sot just hame

Monrie not-eyes areaning

Having thee it alere

Dum-dey monethe-kind glowingover me time of 1 so cleum-diddley money-of-girl like the, he your life arly)

An, to be $y$

must b-you'd-me neon lwas tr

See that gswer me our fiddle funnyarry

Thaights

Yelying tirl, watchsincerele

I think

in thet's me

Tlow eyes,o expl that sceny

(Answethen maybe rich mhere's a the spotain the

Diggin' $r$ me sin you'd seean's wo speciallights ofe lawsthe dancincerely) me, baby rld mon love

Li the city of geg queen

Di

Was it a

You'd be mey moneke an ea nights

Iometryggin' the dream, ine

And wey moneygle flyi am behin

And Idancing qua lie?

L'd be toge

alwaysng with d you, I' couldeen

ike reftther all $t$ sunny a dove

Ill alwaysn't heections he time

$\mathrm{Wi}$

in the r'll find find youlp it of your sh I was, ich man' it in t, I am th

I justmind, mydum-dum-dis world he end

Ife tiger

$\mathrm{P}$ had t love, mddle, your

ahaaa 
al I keep oeople whoo kissy life

Ar darling $f$ the thn searchi fear me the te the woriddle

ings I cng, but unever go eacherds you trould do ntil thennear me,

One oy to find

if I had

I'm CarrI am the $f$ thes, my love a littlie not-thtiger

And e days, my lifee money e-kind-ofif I meet

Gonn

But I kn

it's a r-girl-youyou, what tell ow I don'ich man"d-marry if I eat yhim I t possesss world

That's meou, I am tdream you

So g

Money mo

There's he tiger

Iof himo away, Gney monea special am behind everyod bless y

must be love

Lik you, I'll nightyou

You a funny

ine an eagl always fi

One ofre still the riche flying nd you, I these my love a man's wowith a doam the tigdays

Gond my lifrld

moneyve

I'll fier

Tiger, nna shoe

Yes I kn money mond it in ttiger, tigw him Iow I don'tney

alwayhe end

If er!

care

$\mathrm{G}$ possess ys sunny

iI keep on onna teou

So go an the ricsearching, ach himway, God bh man's w but until you a less you

Yorld

ahaaa then

I'm lesson ou are sti

all the tCarrie notalrightll my lovehings I co-the-kind-

What a and my liuld do

if of-girl-yo crazy fe

Still $\mathrm{mI}$ had a liu'd-marry day

Whey one and ttle money

That's me

n I kisonly 
it's a rised thech man's w teacheorld

It's $r$

All mya rich man sense h's world

ad flown away

When I kissed the teacher

My whole class went wild

As I held my breath, the world stood still, but then he just smiled

I was in the seventh heaven

When I kissed the teacher

(I wanna hug, hug, hug him)

When I kissed the teacher

(I wanna hug, hug him)

When I kissed the teacher

(I wanna hug, hug, hug him)

When I kissed the teacher

(I wanna hug, hug him)

When I kissed the teacher

(I wanna hug, hug, hug him) 
Ryan Jordan

What is noise (music) to you?

Noise music is the freedom to express oneself sonically without the constraints of existing musical structure, tradition, or genre. It also acts as a gateway to many other areas of interest including art, performance, science, philosophy, society, etc., taking the creator away from just musical study. Sound is merely an output of the process.

\section{Why do you make it?}

Because it is easy. 
NOISE IN AND AS MUSIC 